\title{
Plasma Fluctuations, Local Partial Saha Equilibrium, and the Broadening of Vacuum-Arc Ion Charge State Distributions
}

\author{
André Anders, Senior Member, IEEE
}

\begin{abstract}
Charge state distributions (CSD's) of $\mathrm{Fe}, \mathrm{Co}, \mathrm{Zr}, \mathrm{Nb}$, $\mathrm{Hf}, \mathrm{Ag}, \mathrm{Pt}, \mathrm{Au}$ ions produced at vacuum arc cathode spots are investigated without often-used averaging procedures. Statistical results are used to evaluate the contribution of fluctuations to the broadening of averaged CSD's. Calculations of Saha equations in the Debye-Hückel approximation are often successful in replicating experimental CSD's even when using the simple model of "instantaneous freezing." This allows us to use charge state spectrometry as a plasma diagnostic tool for the dense plasma near the cathode spot. Typically, total heavy particle (atoms and ions) densities and electron temperatures of $10^{24}-10^{25} \mathrm{~m}^{-3}$ and 3-4 eV have been measured this way at the transition zone from equilibrium to nonequilibrium, the "event horizon." It was also found that CSD's for some elements such as Co, Ag, and Hf could not be accurately simulated with the assumption of instantaneous freezing. Here, a more gradual transition to nonequilibrium occurs. This transition can be described by the model of partial local Saha equilibrium. Finally, an axial magnetic field was found to soften the transition to nonequilibrium thus causing broadening of experimental CSD's.
\end{abstract}

\section{INTRODUCTION}

$\mathbf{T}$ HE VACUUM arc plasma is produced at cathode spots-minuscule locations of very high current density and plasma density [1]. The plasma is usually fully ionized and contains multiple ion charge states. The charge state distributions (CSD's) of ions of vacuum arc plasmas have been extensively studied using time-of-flight charge-tomass spectrometry [2]-[12]. The most complete table of experimental CSD's is given in [10] for 50 cathode materials. CSD's of multispecies plasmas (from alloy cathodes) have been investigated experimentally [13], [14] and theoretically [15]. Furthermore, it has been found that ion charge states can be enhanced by external magnetic fields [7], [8], [16] and by high discharge currents [11].

Over the past decades, several attempts have been made to calculate CSD's near the cathode under equilibrium conditions using the well-known Saha equations [12], [17]-[20]. Baksht et al. [17] spectroscopically investigated nanosecond discharges of aluminum in vacuum and found that a transition to nonequilibrium occurs rapidly when the plasma expands into the vacuum ambient. Pustovit and coworkers [12] used mass spectrometry for In, Ga, and Sn cathodes; their conclu-

Manuscript received November 19, 1998; revised March 17, 1999. This work was supported by the U.S. Department of Energy under Contract DEAC03-76SF00098.

The author is with the Lawrence Berkeley National Laboratory, University of California, Berkeley, CA 94720 USA (e-mail: aanders@lbl.gov).

Publisher Item Identifier S 0093-3813(99)06880-0. sions are in agreement with the physical picture established by Baksht et al. [17]. Radic and Santic [18] and Hantzsche [20] used the Saha approach to determine reasonable ranges of density and temperature near the cathode spot for $\mathrm{Cd} \mathrm{Mg}$, $\mathrm{Al}, \mathrm{Ni}, \mathrm{Mo}$, and $\mathrm{Cu}$. All authors found high density values (of order $10^{26} \mathrm{~m}^{-3}$ ) and temperature values up to $5 \mathrm{eV}$. Interestingly, although all of the investigators concluded that there is reasonable agreement between experiment and theory, they pointed out that further research is needed. Radic and Santic as well as Hantzsche noted that the Saha-determined temperatures are higher for higher-charged ions than for just one- or double-charged ions.

In a previous paper [21], the formation of ions in vacuum arc discharges has been calculated for all metallic elements of the Periodic Table based on the assumptions that: 1) local thermodynamic equilibrium (LTE) can be assumed in the vicinity of cathode spots [22], 2) Saha equations of weakly nonideal plasmas (Debye-Hückel approximation) describe the CSD's correctly as long as equilibrium is valid, 3) CSD's remain constant (they "freeze") when the plasmas expand into the vacuum and become non-LTE plasmas, and 4) the fluctuations of plasma temperature and density at freezing are small enough to allow the introduction of a single "effective freezing temperature" in combination with a single "effective freezing density" for each element. Using this approach, not only experimental CSD data could be replicated but CSD's of not-yet-used cathode elements were predicted [21]. Meanwhile, the CSD of one of the previously not-used elements, boron, has been measured and very good agreement with the predictions has been found [23].

In fact, good agreement between experimental and simulated CSD's was obtained for most elements except a group of elements for which the experimentally observed CSD's are substantially broader than the calculated. This group includes, among others, Mo, Ag, Hf, Ta, W, and Ir. Obviously, not all assumptions are well justified for these elements. In this paper we concentrate on the issue of CSD broadening by investigating the effect of plasma fluctuations, and by replacing the concept of "instantaneous freezing" by the concept of "stepwise freezing": the model of partial local Saha equilibrium (PLSE) is introduced in analogy to partial local thermodynamic equilibrium (PLTE), which is well-known in plasma spectroscopy.

The rest of the paper is organized as follows. In Section II, the calculation of equilibrium CSD by Saha equations is briefly 
described. The simple model of "instantaneous freezing" and its limitations are explained in Section III. To illuminate the role of plasma fluctuations, experiments have been performed which are described in Section IV. The model of partial local Saha equilibrium (PLSE) is introduced in Section V, and the effect of an axial magnetic field is discussed in Section VI.

\section{SAHA EQUILIBRIUM}

Ion charge state distributions are the result of inelastic, ionizing or recombining collisions of electrons with "heavy" plasma particles, i.e., neutral atoms, singly charged ions, doubly charged ions, etc. In equilibrium, collisions are sufficiently frequent so as to cause numerous ionizing and recombining events per unit time. As a result, the ionization rate equals the recombination rate and thus the ratios between the fractions of various charged particles become time-independent. The extremely dense spot plasma provides the conditions for equilibrium (sufficiently frequent collisions). Ionization and recombination events can be theoretically considered as chemical reactions, and the corresponding steady-state reaction rate equations are known as Saha equations [24]-[26]. If the ion CSD is fully described by Saha equations, the plasma is said to be in Saha equilibrium.

The Saha equations have the form

$$
\begin{aligned}
\frac{n_{e} n_{Q+1}}{n_{Q}}= & S_{Q}(T) \\
= & \Lambda_{B}^{-3} \frac{2 \Sigma_{Q+1}(T)}{\Sigma_{Q}(T)} \exp \left(-\frac{E_{Q}-\Delta E_{Q}}{k T}\right) \\
& \quad Q=0,1, \cdots Q_{\max }
\end{aligned}
$$

where $n_{e}$ and $n_{Q}$ are the densities of free electrons and ions of charge state $Q$, respectively, $Q=0$ for neutral atoms, $Q=1$ for singly ionized ions, etc., with $Q_{\max }$ the maximum charge state abundant in the plasma; $\Sigma_{Q}(T)$ is the temperaturedependent partition function of ions of charge state $Q, k$ is the Boltzmann constant, $T$ is the temperature

$$
\Lambda_{B}=h /\left(2 \pi m_{e} k T\right)^{1 / 2}
$$

is the thermal De Broglie wavelength, and $E_{Q}$ is the ionization energy of the $Q$-fold charged ion. The lowering of the ionization energy $\Delta E_{Q}$ reflects the particle interaction or nonideal nature of the plasma. For weakly nonideal plasmas, the lowering of the ionization energies can be described by the relatively simple Debye-Hückel theory [25]

$$
\Delta E_{Q}=\frac{(Q+1) e^{2}}{4 \pi \varepsilon_{0}\left(\lambda_{D}+\Lambda_{B} / 8\right)}
$$

where

$$
\lambda_{D}=\left[\varepsilon_{0} k T / e^{2}\left(n_{e}+\sum_{Q} Q^{2} n_{Q}\right)\right]^{1 / 2}
$$

is the generalized Debye length, and $\varepsilon_{0}$ is the permittivity of vacuum. The lowering of the ionization energy can be neglected for expanded plasmas but is significant for dense plasmas. The Debye-Hückel approximation becomes invalid when the plasma is extremely dense ( $\Delta E_{Q}$ is comparable to $\left.E_{Q}\right)$.

The partition function reflects the structure of the electron shells and is defined by the equation [27]

$$
\Sigma_{Q}(T)=\sum_{s=1}^{s=s_{\max }} g_{Q, s} \exp \left(-\frac{E_{Q, s}}{k T}\right)
$$

where $s$ is the level index ( $s=1$ is the ground state, $s_{\max }$ is the highest excited level which is bound), $g_{Q, s}$ and $E_{Q, s}$ are the statistical weight and the energy of level $s$ of an ion with charge state $Q$, respectively. The statistical weight $g_{Q, s}=2 J_{Q, s}+1$ can be obtained using the total angular momentum $J_{Q, s}$ given in spectral tables. The system of (1)-(5) is completed by the charge-neutrality condition

$$
n_{e}=\sum_{Q}^{Q_{\max }} Q n_{Q}
$$

The percentages of ion charge states are expressed as particle fractions of the total ion density (i.e., without neutrals)

$$
f_{Q}=\frac{n_{Q}}{\sum_{Q^{\prime}=1}^{\prime} n_{Q^{\prime}}} \cdot 100 \%
$$

to allow comparison with experimental time-of-flight results. The mean ion charge state is defined by

$$
\bar{Q}=\sum_{Q=1}^{Q_{\max }} Q n_{Q} / \sum_{Q=1}^{Q_{\max }} n_{Q}
$$

The numerical calculations were done as follows. An initial neutral atom density and start electron density were assumed and the set of Saha equations was solved in a first iteration step, resulting in a temporary electron density which most likely was different from the preset start electron density. The assumed neutral density was then varied to minimize the difference between the preset and calculated electron density until a selfconsistent CSD was obtained. The calculation was continued with the same temperature at the next electron density (2\% greater than the previous electron density). The calculation was stopped at high densities when the lowering of the ionization energy approached the value of the ionization energy itself. This is the region of strongly nonideal plasma where the Debye-Hückel theory breaks down. The whole procedure was then repeated for the next preset temperature, until the desired range of plasma parameters had been covered. For numerical data on ionization energies and partition functions see [21].

\section{Model OF InstantaneOUS FreEZING}

The plasma density in the micrometer-size vicinity of a cathode spot is at least of order $10^{26} \mathrm{~m}^{-3}$ [28]. It can be shown that this extremely high value allows the establishment of Saha equilibrium even if the residence time of an ion in this region is very short (nanoseconds). Strong density gradients cause rapid plasma expansion into the vacuum ambient, and as the density decreases, the frequency of ionizing and recombining collisions decreases also. There is a transition from Saha 


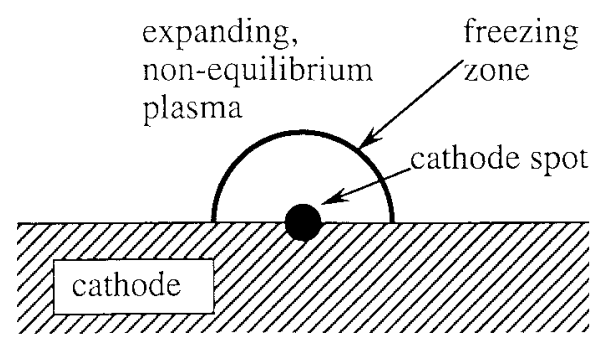

Fig. 1. Schematic illustration of the cathode spot, the freezing zone, and the nonequilibrium plasma.

equilibrium near the spot to nonequilibrium far from the spot. In the simple model of "instantaneous freezing," the extension of this transition zone is infinitesimally small (Fig. 1).

A single temperature and density can be associated with the transition at any given time, but this temperature and density may fluctuate in time. The ion CSD is established inside the volume defined by the transition zone. It freezes (i.e., stays constant afterwards) when the ions go through the transition zone. Observable CSD's are therefore "fingerprints" of the plasma conditions at the transition zone. One can also consider the transition zone as an "event horizon" beyond which we cannot probe the plasma using CSD information. CSD's are understood as relicts of microexplosions. We realize that the concept of "instantaneous freezing" is equivalent to a sharp, well-defined event horizon. Previous work [21] showed that the event horizon is at high densities of order $10^{24}-10^{25}$ $\mathrm{m}^{-3}$, that is, at very small distance from the cathode spot center (of order $100 \mu \mathrm{m}$ ). Therefore, observable CSD's carry information from a region very close to the cathode spot, and CSD spectrometry turns out to be a powerful tool for learning about cathode spot plasma parameters and processes.

\section{Plasma AND CSD Fluctuations}

Published data of experimental CSD's (e.g., [6], [10], [29]) have been determined by averaging over many individual time-of-flight (TOF) data samples (usually one sample per one pulsed vacuum arc). It is known that cathode spots are of nonstationary nature (that is true for both pulsed and DC arcs), and a CSD sampled at a certain instant of time differs from a CSD measured at a different time even when macroscopic conditions are kept constant. For instance, in earlier work [7] it was shown that CSD samples scatter when individually measured for a $150 \mathrm{~ns}$ gate $100 \mu \mathrm{s}$ after discharge triggering. This scattering suggests that the plasma parameters of the cathode spot and event horizon fluctuate-a well-known phenomenon of vacuum arcs. It is straightforward to expect that experimentally determined CSD's are broadened by the averaging procedure, as suggested in [21]. This may (or may not) be particularly pronounced for the "problematic" elements such as Co, Mo, Ag, Hf, Ta, W, and Ir.

In order to experimentally verify this hypothesis, hundreds of individual CSD's have been measured without averaging. The same TOF equipment [30] was used as in previous studies [6], [10], [29]. The gate duration was always $150 \mathrm{~ns}$ full width at half maximum and determined the time resolution of these CSD measurements. Three groups of cathode materials were
TABLE I

Statistical Data of Ion Charge States, Derived From 100 CSD's MeAsurements PER ElEMENT. The Data with Magnetic Field Are Discussed in Section VI

\begin{tabular}{lllll}
\hline & $Q$ (mean) & $Q(\max )$ & $\mathrm{Q}(\mathrm{min})$ & $\begin{array}{l}\text { Standard } \\
\text { Deviation }\end{array}$ \\
\hline $\mathrm{Ag}$ & 1.9301 & 2.165 & 1.705 & 0.1056 \\
$\mathrm{Pt}$ & 2.0409 & 2.151 & 1.876 & 0.0545 \\
$\mathrm{Au}$ & 1.9684 & 2.052 & 1.911 & 0.0302 \\
$\mathrm{Fe}$ & 1.8796 & 1.974 & 1.722 & 0.0475 \\
$\mathrm{Co}$ & 1.8369 & 1.989 & 1.615 & 0.0707 \\
$\mathrm{Zr}$ & 2.5297 & 2.743 & 2.247 & 0.0828 \\
$\mathrm{Nb}$ & 2.8463 & 3.093 & 2.486 & 0.1098 \\
$\mathrm{Hf}$ & 1.7078 & 2.160 & 1.344 & 0.1506 \\
$\mathrm{Zr}$ (B-field) & 3.5921 & 3.804 & 3.170 & 0.1482 \\
$\mathrm{Nb}$ (B-field) & 4.1232 & 4.275 & 3.710 & 0.0904 \\
$\mathrm{Hf}$ (B-field) & 2.8691 & 3.094 & 2.621 & 0.0905 \\
\hline
\end{tabular}

selected for this study: 1) Fe, Co; 2) Zr, Nb, Hf; and 3) Ag, Pt, $\mathrm{Au}$. The elements in these groups are neighbors in the Periodic Table but behave very differently in terms of CSD broadening [21]. For instance, Au can be well associated with a single effective plasma temperature and density at the event horizon (where the CSD freezes), while Ag and Pt show broadening. Similarly, $\mathrm{Fe}$ is much better approximated than $\mathrm{Co}$, and $\mathrm{Zr}$ better than $\mathrm{Nb}$ and $\mathrm{Hf}$.

The following parameters were used: arc current $300 \mathrm{~A}$, arc duration $250 \mu \mathrm{s}$, arc repetition rate $5 \mathrm{~Hz}$, TOF gate position $150 \mu \mathrm{s}$ after arc triggering, 3-grid ion extraction system with $-3 \mathrm{kV}$ suppressor voltage and $+40 \mathrm{kV}$ extraction voltage. The vacuum base pressure was $4 \times 10^{-6}$ torr before and about 1 $\times 10^{-5}$ torr during arc operation. A relatively high arc pulse repetition rate was chosen to minimize the effect of cathode gas load [31]. The arc was operated for about $5 \mathrm{~s}$ before data was taken in a "snapshot" mode via a fast digital storage oscilloscope. After data acquisition was completed, the arc was run for the next $5 \mathrm{~s}$ and the next data snapshot taken. By following this procedure, arc operation on cleaned cathodes as well as single-shot data acquisition was accomplished.

Fig. 2 shows the result of the experiment for the group of $\mathrm{Ag}, \mathrm{Pt}$, and $\mathrm{Au}$. We see clearly that gold has the smallest scattering of data while the data for silver scatter significantly. This is exactly as hypothesized by the broadening behavior although there is no obvious physical explanation at this point.

The same experiments for the other elements of the other two groups give somewhat similar results but the difference between the elements is not as great as expected (see Table I). In particular, the difference of scattering for $\mathrm{Fe}$ and $\mathrm{Co}$ is small, indicating that there must be another broadening mechanism (discussed later in this paper).

Let us investigate the fluctuation of those elements whose CSD can be well approximated by a single temperature and density combination. Here, the concept of instantaneous freezing is a good approximation, delivering the plasma parameters at the event horizon. As an example, we show the results for gold and iron and in Fig. 3, we see that temperature and density are correlated. 


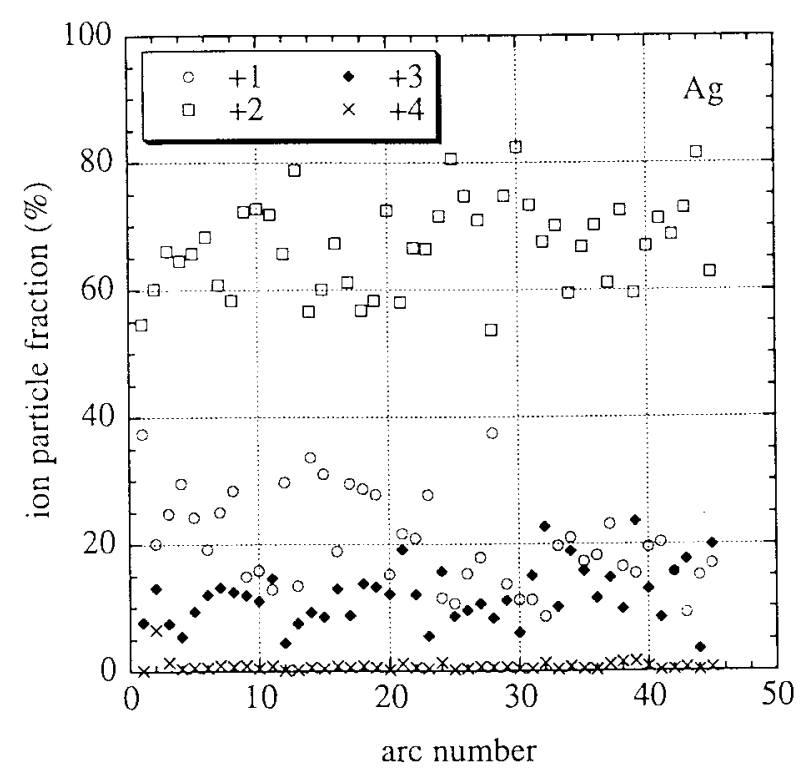

(a)

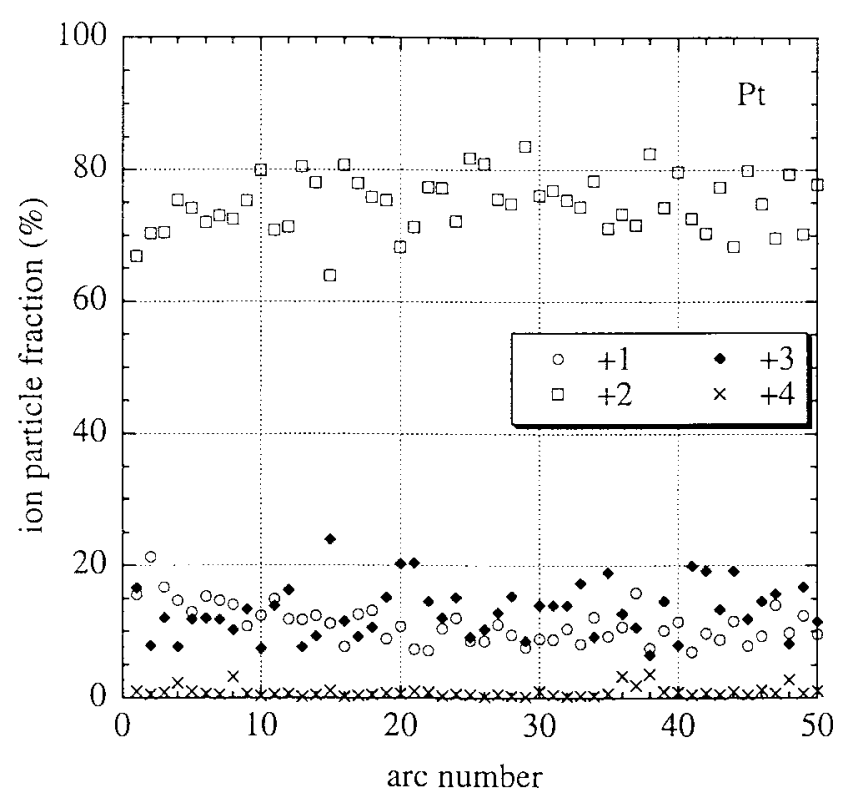

(b)

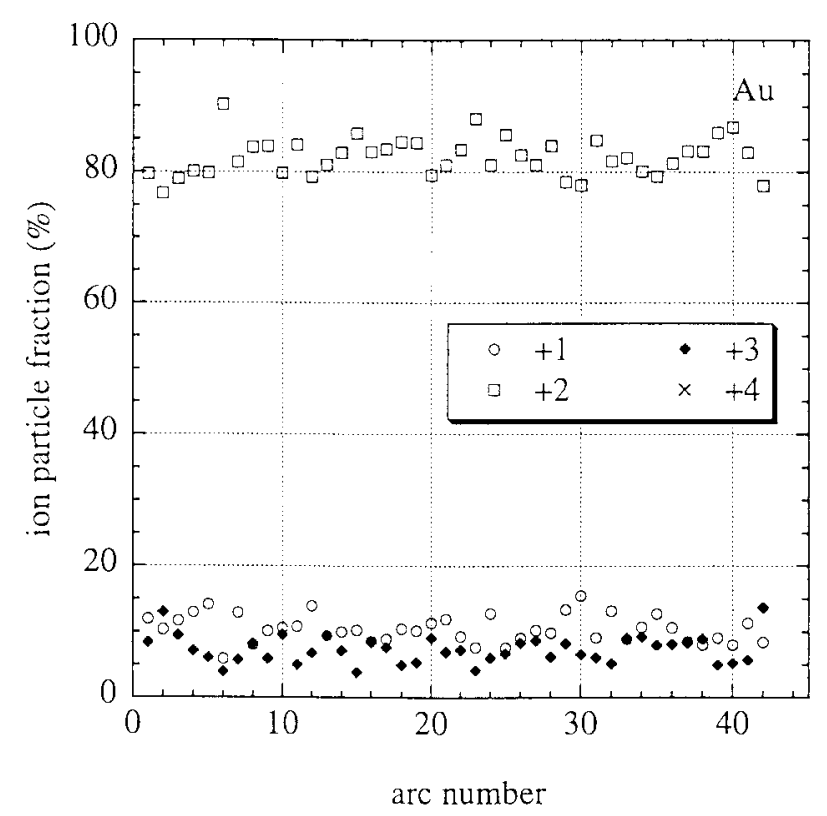

(c)

Fig. 2. Statistical CSD data for: (a) silver, (b) platinum, and (c) gold.

A plausible but unproved interpretation is that if momentarily a high power density is invested in the plasma, the plasma is hotter, creating stronger gradients which in turn accelerate the plasma expansion, which shortens the characteristic plasma expansion time. A shorter plasma expansion time causes freezing at shorter characteristic inelastic collision times (the onset of nonequilibrium is faster), and thus freezing happens at higher density. This would allow probing deeper into the spot plasma, i.e., closer to the spot center.

In any case, CSD data reflect the change of plasma parameters in a very sensitive way. Since each data set was taken with a gate time of $150 \mathrm{~ns}$, TOF data give a very good time resolution in terms of plasma diagnostics. Looking at individual
CSD data allows statistical analysis of the nonstationary nature of the spot with about $150 \mathrm{~ns}$ resolution. The data suggest that there are instants of higher and lower power input into the spot, which is in agreement with fluctuations seen in voltage and radiation.

By analyzing individual CSD data, it could readily be seen that some elements give data which cannot be approximated by a single temperature and density combination, although the averaged data gave reasonable, approximate effective values. These elements have been marked with label "B" (B stands for "broadened") in the Periodic Table [21]. If the averaging procedure was responsible for the broadening of their CSD, individual CSD data should be at least approximately repli- 


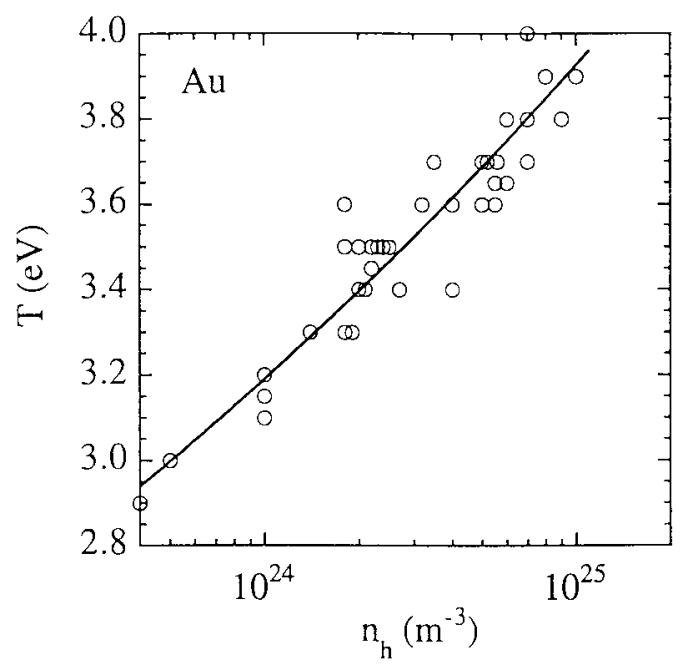

(a)

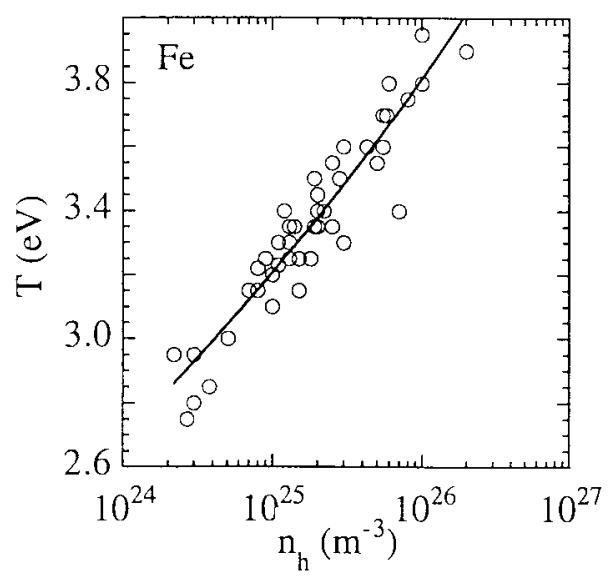

(b)

Fig. 3. Heavy particle density and plasma temperature correlation at the freezing zone (event horizon): (a) gold and (b) iron plasma. Accuracy of data: about $25 \%$ and $0.05 \mathrm{eV}$ for density and temperature, respectively.

cated by the simulations. However, this is not the case for some elements such as $\mathrm{Co}, \mathrm{Nb}$, and $\mathrm{Hf}$. Consequently, there must be an another broadening mechanism which is not well described by the model of instantaneous freezing.

\section{Partial Local Saha Equilibrium}

It is clear that freezing does not happen instantaneously but the transition zone has a finite thickness. The reason why the model of instantaneous freezing worked so well for a number of elements is that the thickness of the transition zone turned out be negligibly thin (the event horizon is "sharp"). In this section we will drop this assumption and consider a diffuse transition zone. Unfortunately, this will blur the clear picture we had of the local plasma parameters at the event horizon.

It is intuitively clear that the Saha equilibrium is gradually lost when transitioning to nonequilibrium. A complete description of the process can be achieved by solving the system of coupled rate equations with the collisional-radiative ionization and recombination coefficients in conjunction with the hydrodynamic equations and energy balance. This is a formidable

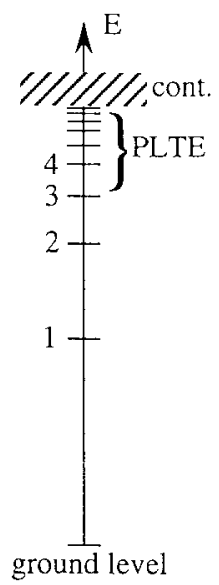

(a)

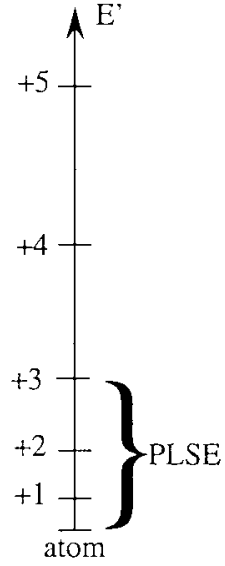

(b)
Fig. 4. (a) Schematic of the energy level system of bound states of an atom or ion and (b) schematic of the system of ionization energies.

task. Some physical insight into the ionization/recombination processes can be gained by the much simpler model of partial local Saha equilibrium (PLSE) which in introduced below.

The model of partial local thermodynamic equilibrium (PLTE) is well-known in plasma spectroscopy. Griem [32] defines a homogeneous, steady-state plasma system in complete thermodynamic equilibrium (CTE) when distributions of the various bound states and ionization stages are in equilibrium (i.e., described by Boltzmann-Saha distributions) and the velocities of atoms, ions, and electrons are determined by Boltzmann distributions of the same temperature. Such a system can be characterized by a small number of parameters. The parameters may change gradually in space and time, which leads to the model of complete local thermodynamic equilibrium (CLTE).

Unfortunately, real plasma systems are rarely in CLTE, and thus more complicated models must be employed. One straightforward approach is to consider only a part of the system, for instance levels of optical transitions when performing plasma spectroscopy. Here, the relation between the electron population densities of the various bound states of an atom or ion is of interest. The energy level structure of bound states of an atom or ion is shown schematically in Fig. 4(a). As a rule, the largest energy gap is between the ground state and the first excited state, with decreasing energy gaps when approaching the electron continuum. It has been shown that all energy levels are in equilibrium if the electron density is sufficiently high (see, for instance, [24], [26]). If the electron density decreases gradually, the energy level with the largest gap (usually the ground state) will not have enough collision events causing population and depopulation, thus this level will not be in equilibrium with the remaining system. The remaining system, excited bound states and electron continuum, are still in what is called partial local thermodynamic equilibrium (PLTE). With further decreasing electron density, more-and-more levels will not be in PLTE, until only the highest, closely-spaced levels remain in PLTE with the electron continuum.

If the velocity distribution of the colliding particles is thermal (Boltzmann distribution), and if collisional processes 
are more important than radiative decay and recombination, the principle of detailed balancing can be used to find a steady-state solution to the rate equations. According to this principle, one can balance the forward and reverse reactions caused by collisions. As a result, the PLTE model allows the derivation of a modified Boltzmann formula for excited states and a modified Boltzmann-Saha equation which relates the density of ions with charge state $Q$ to the upper levels of ions with charge state $Q-1$ [26]. The modified Boltzmann-Saha equations will not serve our purpose of relating the ratios of ions since it excludes ions which are in low level states.

For charge-state spectrometry, the principle of detailed balancing can be used to consider ionization and recombination reactions in analogy to the consideration of excitation and deexcitation reactions in optical spectroscopy. The PLTE concept allowed the calculation of population ratios for selected excited states in the absence of CLTE. Analogously, the new concept of PLSE will allow calculation of the ratio of charge states in the absence of complete Saha equilibrium.

The ionization-recombination balance equations are

$$
\begin{array}{rr}
\frac{\partial n_{Q}}{\partial t}= & n_{Q+1} n_{e}^{2} \alpha_{Q+1, Q}-n_{Q} n_{e} \beta_{Q, Q+1} \quad \text { for } Q=0 \\
\frac{\partial n_{Q}}{\partial t}= & n_{Q-1} n_{e} \beta_{Q-1, Q}+n_{Q+1} n_{e}^{2} \alpha_{Q+1, Q} \\
& -n_{Q} n_{e} \beta_{Q, Q+1}-n_{Q} n_{e}^{2} \alpha_{Q, Q-1}
\end{array}
$$

where $\beta$ and $\alpha$ are the ionization and recombination coefficients, respectively. We follow here the notation of Biberman et al. [33] who defines the coefficients in such a way that they are density-independent (except for the nonideal plasma correction). This is convenient since it shows, for instance, that recombination is determined by three-body collisions (inelastic two-body collisions are unlikely to satisfy energy and momentum conservation). According to the principle of detailed balancing, radiative ionization and recombination is neglected. Note that this principle can be applied although the ion velocity distribution is nonthermal; electrons are Maxwellian (thermal) and much faster than ions, thus the relative collision velocity is very close to thermal. The first two terms on the right-hand side of (9b) describe the increase of ion density of charge state $Q$ by ionization (from $Q-1$ ) and recombination (from $Q+1$ ), and the other two terms describe the decrease by ionization (to $Q+1$ ) and recombination (to $Q-1$ ). From (9b), the characteristic time of change by ionization and recombination reactions is shown at the bottom of the page in (10). As long as the plasma is in Saha equilibrium (near the spot), we can use the Saha factors $S_{Q}$ defined in (1) and obtain that shown at the bottom of the page in (11).

From this expression we can easily derive the characteristic times of the individual processes and their dependence on the charge state $Q$ if we know the charge-state dependence of the ionization and recombination coefficients. Following Biberman [33], and taking into account the energy correction for nonideal plasmas, we have

$$
\begin{aligned}
\beta_{Q, Q+1}\left(E_{Q}, T\right) & \sim \frac{\Sigma_{Q+1}}{g_{Q, q}} \exp \left(-\frac{E_{Q}-\Delta E_{Q}}{k T}\right) /(k T)^{3} \\
\alpha_{Q, Q-1} & \sim(k T)^{-9 / 2}
\end{aligned}
$$

Using these expressions, we see that the first term in the denominator of (11) is independent of $Q$, the second and third terms are proportional to $\exp \left(-\left(E_{Q}-\Delta E_{Q}\right) / k T\right)$, and the last term is independent of $Q$. We can conclude as long as Saha equilibrium applies, the characteristic times for recombination to charge state $Q$ and for ionization from charge state $Q$ increase with $Q$. Therefore, ions of higher charge will tend to be in nonequilibrium sooner than ions of low charge state.

When we apply this result to the expanding spot plasma we realize that the highest ion charge states (such as +5 and +4 ) will leave the Saha equilibrium system first, while lower charge states (such as $+3,+2,+1$, and 0 ) are still in equilibrium. This situation of partial local Saha equilibrium (PLSE) is illustrated in Fig. 4(b). With decreasing density and temperature, the states $+3,+2,+1$ will drop out of PLSE, and freezing is completed. The event horizon (freezing zone) now has a finite thickness because charge states do not freeze simultaneously but successively. This explains why we cannot find a single density-temperature combination in the Saha equations which replicates the experimental CSD for some elements. Because density and temperature cover a range of values, more information than the CSD is required to find unique solutions. A quantitative analysis of PLSE has, for instance, to deal with the energy balance, i.e., the rate the temperature drops while the plasma is expanding. Additional information could come from high-resolution density and temperature measurements or a self-consistent solution of a plasma model.

It is possible, however, to test the concept of PLSE by comparing Saha calculations for different temperatures with experimental CSD data. For this purpose, a CSD database with a step width of $0.05 \mathrm{eV}$ was generated. These data were compared with experimental CSD's. Reasonable ranges of density and temperature can be identified and thus the thickness of the freezing zone can approximately be determined. Fig. 5

$$
\Delta t=\frac{\Delta n_{Q}}{n_{Q-1} n_{e} \beta_{Q-1, Q}+n_{Q+1} n_{e}^{2} \alpha_{Q+1, Q}-n_{Q} n_{e} \beta_{Q, Q+1}-n_{Q} n_{e}^{2} \alpha_{Q, Q-1}}
$$

$$
\Delta t=\frac{\Delta n_{Q}}{n_{Q}} \frac{1}{\frac{n_{e}^{2}}{S_{Q-1}} \beta_{Q-1, Q}+S_{Q} n_{e} \alpha_{Q+1, Q}-n_{e} \beta_{Q, Q+1}-n_{e}^{2} \alpha_{Q, Q-1}}
$$




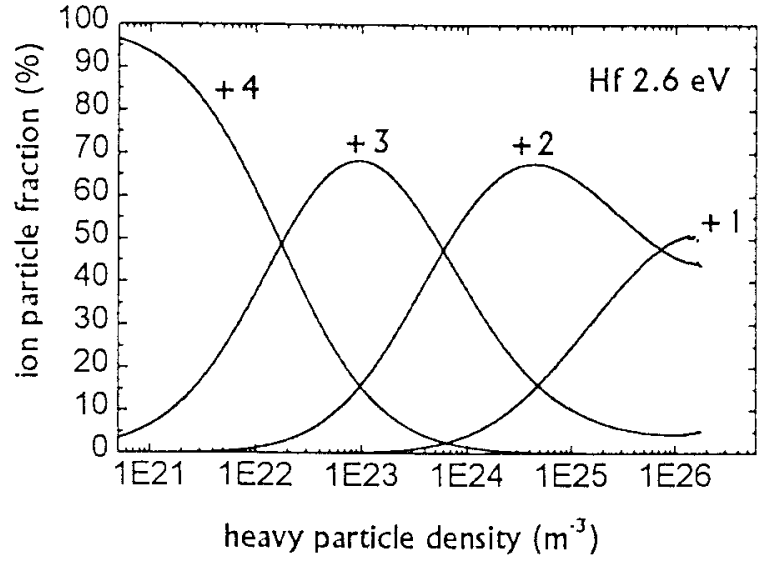

(a)

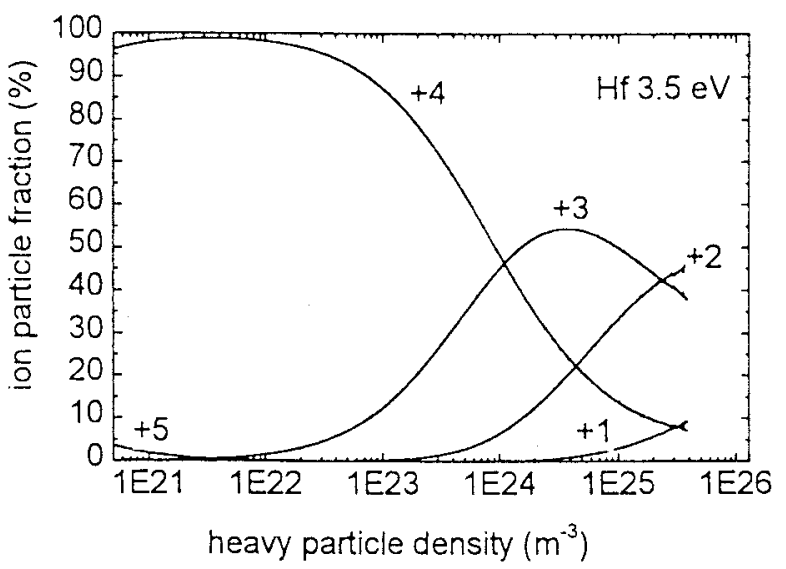

(c)

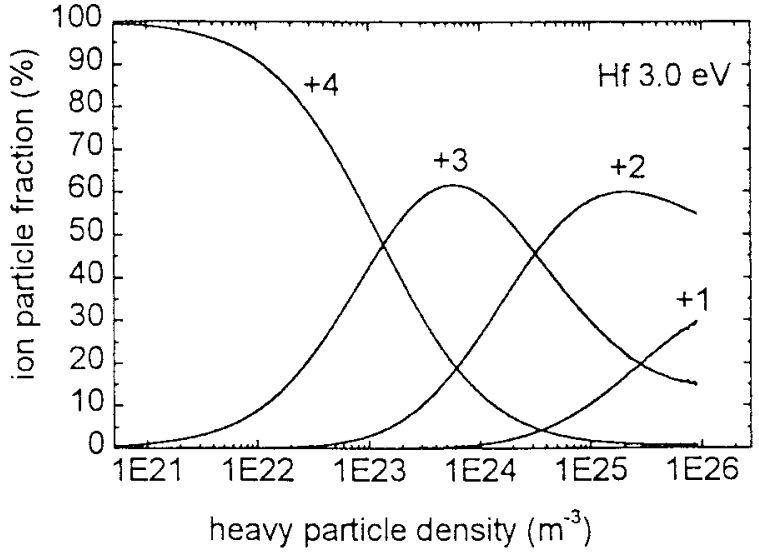

(b)

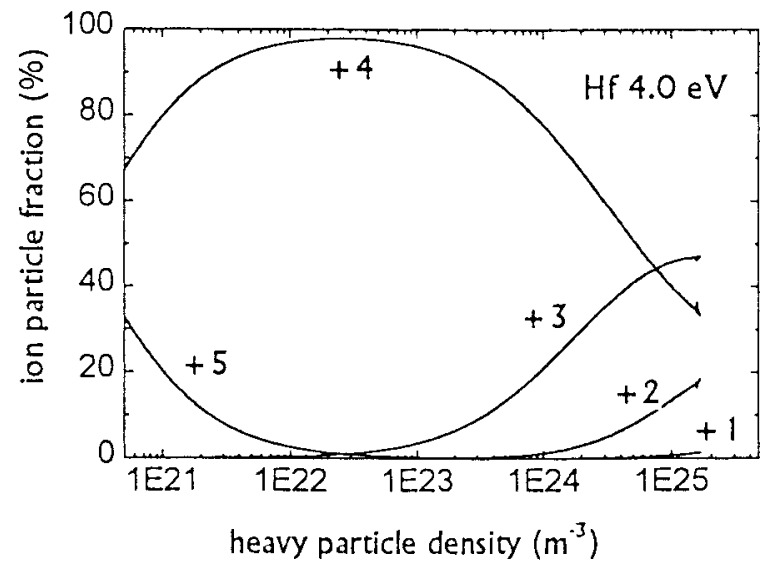

(d)

Fig. 5. Saha calculation of CSD of hafnium at (a) 2.6, (b) 3.0, (c) 3.5, and (d) $4.0 \mathrm{eV}$ (a selection from the calculated Hf data base).

shows four plots from the hafnium data base. All individual hafnium CSD's could be approximated in the temperaturedensity range $3.6 \rightarrow 2.6 \mathrm{eV}$ and $8 \times 10^{25} \mathrm{~m}^{-3} \rightarrow 1 \times 10^{24}$ $\mathrm{m}^{-3}$. Fig. 6 illustrates the finite thickness of the freezing zone in accordance with the PLSE model.

\section{EFFECT OF MAGNETIC FIELD}

In order to assess the effect of an axial magnetic field on the statistics and broadening of CSD's, experiments were conducted with the same parameters as described in Section IV (except the field). The axial magnetic field was obtained by a coil in the cathode-anode discharge region, as described in [29]. The coil current was in series with the arc current generating a maximum field strength of $B=30 \mathrm{mT}(0.1$ mT/A).

As previously observed, CSD's are shifted to higher values. By calculating the particle currents (as opposed to electrical currents) and by statistically analyzing the data for several elements it was found that the standard deviation is increased or reduced compared to the data without magnetic field (see Table I). As already noted in a previous paper [21], experimental CSD's are not only shifted but become broader in the presence of a magnetic field. The present experimental results show that this broadening cannot be attributed to statistical fluctuations and the averaging procedure. Therefore, the other

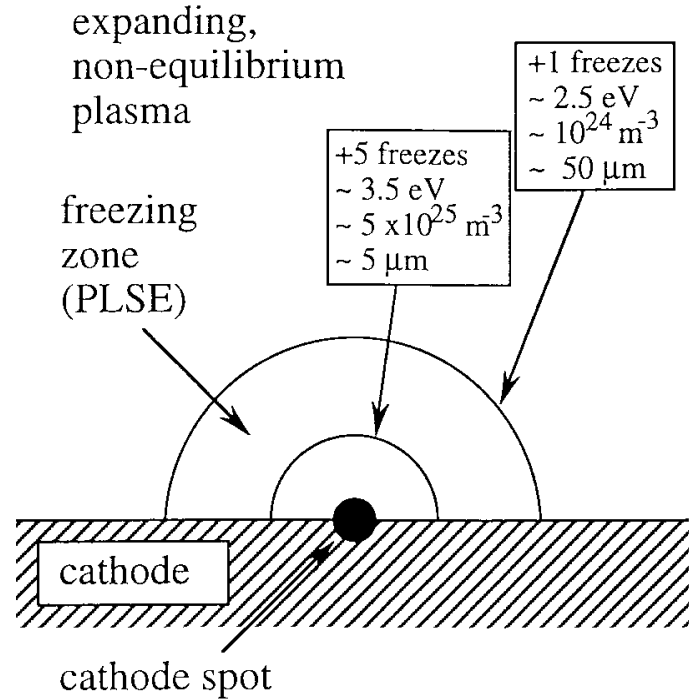

Fig. 6. As Fig. 1, but this time showing the finite thickness of the freezing zone in which stepwise freezing occurs according to the model of partial local Saha equilibrium (PLSE). The numbers are approximate data obtained for hafnium; they are given to illustrate the physical situation including lateral dimensions.

broadening mechanism, stepwise CSD freezing in the PLSE zone, seems to be responsible. This is in agreement with the physical picture of less-rapid expansion in a magnetic 
field. Electrons for the plasma are easily magnetized even at relatively low magnetic fields, and they essentially follow the direction of the magnetic field lines. The plasma appears as a jet, with a reduced density gradient in the direction of expansion. Therefore, expansion is slower and so is the freezing, with the consequences described in Section V.

\section{ACKNOWLEDGMENT}

The author is indebted to T. Schülke for his invaluable help in the numerical solution of the Saha equations.

\section{REFERENCES}

[1] R. L. Boxman, D. M. Sanders, and P. J. Martin, Eds., Handbook of Vacuum Arc Science and Technology. Park Ridge, NJ: Noyes, 1995.

[2] V. M. Lunev, V. G. Padalka, and V. M. Khoroshikh, "Plasma properties of a metal vacuum arc," Sov. Phys. Tech. Phys., vol. 22, no. 7, pp. 858-861, 1977.

[3] W. D. Davis and H. C. Miller, "Analysis of the electrode products emitted by dc arcs in a vacuum ambient," J. Appl. Phys., vol. 40, no. 5, pp. 2212-2221, 1969

[4] A. A. Plyutto, V. N. Ryzhkov, and A. T. Kapin, "High speed plasma streams in vacuum arcs," Sov. Phys. JETP, vol. 20, no. 2, pp. 328-337, 1965.

[5] I. G. Brown, B. Feinberg, and J. E. Galvin, "Multiply stripped ion generation in the metal vapor vacuum arc," J. Appl. Phys., vol. 63, no. 10 , pp. 4889-4898, 1988.

[6] I. G. Brown and X. Godechot, "Vacuum arc ion charge-state distributions," IEEE Trans. Plasma Sci., vol. 19, pp. 713-717, Oct. 1991.

[7] A. Anders, S. Anders, B. Jüttner, and I. G. Brown, "Time dependence of vacuum arc parameters," IEEE Trans. Plasma Sci., vol. 21, pp. 305-311, June 1993.

[8] E. Oks, I. G. Brown, M. R. Dickinson, R. A. MacGill, P. SpKdtke, H Emig, and B. H. Wolf, "Elevated ion charge states in vacuum arc plasmas in a magnetic field," Appl. Phys. Lett., vol. 67, no. 2, pp. 200-202, 1995.

[9] F. J. Paoloni and I. G. Brown, "Some observations of the effect of magnetic field and arc current on the vacuum arc charge state distribution," Rev Sci. Instrum., vol. 66, no. 7, pp. 3855-3858, 1995.

[10] I. G. Brown, "Vacuum arc ion sources," Rev. Sci. Instrum., vol. 65, no. 10, pp. 3061-3081, 1994.

[11] A. Anders, I. Brown, M. Dickinson, and R. MacGill, "High ion charge states in a high-current, short-pulse vacuum arc ion source," Rev. Sci. Instrum., vol. 67, no. 3, pp. 1202-1204, 1996.

[12] A. N. Pustovit, V. I. Zhila, and G. G. Sikharulidze, "Mass spectral diagnostics of plasma formed at cathode tip explosion," Sov. Phys. Tech. Phys., vol. 31, no. 4, pp. 496-497, 1986.

[13] J. Sasaki and I. G. Brown, "Ion spectra of vacuum arc plasma with compound and alloy cathodes," J. Appl. Phys., vol. 66, no. 11, pp. 5198-5203, 1989

[14] J. Sasaki, K. Sugiyama, X. Yao, and I. Brown, "Multiple-species ion beams from titanium-hafnium alloy cathodes in vacuum arc plasmas," J. Appl. Phys., vol. 73, no. 11, pp. 7184-7187, 1993.
[15] T. Schülke and A. Anders, "Ion charge state distributions of alloycathode vacuum arc plasmas," in XVIIIth Int. Symp. Discharges Elect. Insulation Vacuum, Eindhoven, The Netherlands, 1998, pp. 914-917.

[16] I. G. Brown, J. E. Galvin, R. A. MacGill, and M. W. West, "Multiply charged metal ion beams," Nucl. Instrum Meth. Phys. Res. B, vol. 43 no. 3, pp. 455-458, 1989.

[17] R. B. Baksht, A. P. Kudinov, and E. A. Litvinov, "Cathode plasma in the initial stage of a vacuum discharge," Sov. Phys. Tech. Phys., vol. 18, no. 1, pp. 94-97, 1973.

[18] N. Radic and B. Santic, "Composition of vacuum arc plasma," in XIIIth Int. Symp. Discharges Elect. Insulation Vacuum, Paris, France, 1988, pp. 217-219.

[19] S. Anders and A. Anders, "Frozen state of ionization in a cathodic plasma jet of a vacuum arc," J. Phys. D, Appl. Phys., vol. 21, no. 1, pp. 213-215, 1988

[20] E. Hantzsche, "Consequences of balance equations applied to the diffuse plasma of vacuum arcs," IEEE Trans. Plasma Sci., vol. 17, pp. 657-660, Oct. 1989.

[21] A. Anders, "Ion charge state distributions of vacuum arc plasmas: The origin of species," Phys. Rev. E, vol. 55, no. 1, pp. 969-981, 1997.

[22] A. Anders, S. Anders, and E. Hantzsche, "Validity conditions for complete and partial local thermodynamic equilibrium of nonhydrogenic level systems and their application to copper vapor arcs in vacuum," IEEE Trans. Plasma Sci., vol. 17, pp. 653-656, Oct. 1989.

[23] F. Richter, G. Flemming, M. Kühn, S. Peter, and H. Wagner, "Characterization of the arc evaporation of a hot boron cathode," Surf. Coat. Technol., vol. 112, no. 1-3, pp. 43-47, 1999.

[24] H. R. Griem, Plasma Spectroscopy. New York: McGraw-Hill, 1964.

[25] W. Ebeling, W.-D. Kremp, and D. Kraeft, Theory of Bound States and Ionization Equilibrium in Plasmas and Solids. Berlin, Germany: Akademie-Verlag, 1976.

[26] J. Richter, "Radiation of hot gases," in Plasma Diagnostics, W. LochteHoltgreven, Ed. New York: AIP Press, 1995, pp. 1-65.

[27] H.-W. Drawin and P. Felenbok, Data for Plasmas in Local Thermodynamic Equilibrium. Paris, France: Gauthier-Villars, 1965.

[28] A. Anders, S. Anders, B. Jüttner, W. Bötticher, H. Lück, and G Schröder, "Pulsed dye laser diagnostics of vacuum arc cathode spots," IEEE Trans. Plasma Sci., vol. 20, pp. 466-472, Aug. 1992.

[29] E. Oks, A. Anders, I. G. Brown, M. R. Dickinson, and R. A. MacGill, "Ion charge state distribution in high current vacuum arc plasmas in a magnetic field," IEEE Trans. Plasma Sci., vol. 24, pp. 1174-1783, June 1996.

[30] I. G. Brown, J. E. Galvin, R. A. MacGill, and R. T. Wright, "Improved time-of-flight charge state diagnostic," Rev. Sci. Instrum, vol. 58, no. 9, pp. 1589-1592, 1987.

[31] G. Y. Yushkov and A. Anders, "Effect of the pulse repetition rate on the composition and ion charge-state distribution of pulsed vacuum arcs," IEEE Trans. Plasma Sci., vol. 26, pp. 220-226, Apr. 1998.

[32] H. R. Griem, "Validity of local thermal equilibrium in plasma spectroscopy," Phys. Rev., vol. 311, no. 3, pp. 1170-1176, 1963.

[33] L. M. Biberman, V. S. Vorobev, and I. T. Yakubov, Kinetics of the Non-Equilibrium and Low-Temperature Plasma (in Russian). Moscow, Russia: Nauka, 1982

André Anders (M'94-SM'98), for a photography and biography, see this issue, p. 914. 